\title{
Prediction of Human Distribution Volumes of Compounds in Various Elimination Phases Using Physiologically Based Pharmacokinetic Modeling and Experimental Pharmacokinetics in Animals ${ }^{\text {『 }}$
}

\author{
Hidetoshi Shimizu, Kosuke Yoshida, Tomohisa Nakada, Koki Kojima, Akihito Ogasawara, \\ Yoshinobu Nakamaru, and $\odot$ Hiroshi Yamazaki \\ Mitsubishi Tanabe Pharma Corporation, Toda, Saitama, Japan (H.S., K.Y., T.N., K.K., A.O., Y.N.); and Showa Pharmaceutical
University, Machida, Tokyo, Japan (H.Y.)
}

Received July 23, 2018; accepted November 8, 2018

\section{ABSTRACT}

Predicting the pharmacokinetics of compounds in humans is an important part of the drug development process. In this study, the plasma concentration profiles of 10 marketed compounds exhibiting two-phase elimination after intravenous administration in humans were evaluated in terms of distribution volumes just after intravenous administration $\left(V_{1}\right)$, at steady state $\left(V_{s s}\right)$, and in the elimination phase $\left(V_{\beta}\right)$ using physiologically based pharmacokinetic (PBPK) modeling implemented in a commercially available simulator (Simcyp). When developing human PBPK models, the insight gained from prior animal PBPK models based on nonclinical data informed the optimization of the lipophilicity input of the compounds and the selection of the appropriate mechanistic tissue partition methods. The accuracy of $V_{1}, v_{\mathrm{ss}}$, and $V_{\beta}$ values predicted that using human PBPK models developed in accordance with prior animal PBPK models was superior to using those predicted using conventional approaches, such as allometric scaling, especially for $V_{1}$ and $V_{\beta}$. By conventional approaches, the $V_{1}$ and $V_{\beta}$ values of 4-5 of $10 \mathrm{com}-$ pounds were predicted within a 3-fold error of observed values, whereas $V_{\mathrm{ss}}$ values for their majority were predicted as such. PBPK models predicted $V_{1}, V_{\mathrm{ss}}$, and $V_{\beta}$ values for almost all compounds within 3-fold errors, resulting in better predictions of plasma concentration profiles than allometric scaling. The distribution volumes predicted using human PBPK models based on prior animal PBPK modeling were more accurate than those predicted without reference to animal models. This study demonstrated that human PBPK models developed with consideration of animal PBPK models could accurately predict distribution volumes in various elimination phases.

\section{Introduction}

Predicting human pharmacokinetics is an important part of the drug development process. Pharmacokinetics inform the estimation of potential therapeutic doses and safety margins before first-in-human studies. Among the pharmacokinetic parameters, the volume of distribution is a key determinant of the effective half-life $\left(t_{1 / 2}\right)$ and dosing interval of a prospective drug. Many approaches for predicting volumes of distribution have been proposed and are used (Sui et al., 2008). The most commonly used approaches predict human distribution volumes of drugs from animal data via interspecies scaling techniques, such as allometric scaling. The main assumption of allometric scaling is that the factors governing the pharmacokinetics of a drug should scale in relation to body size (Boxenbaum, 1982). Allometric scaling of the unbound volume of distribution has been also reported, because one of the main factors governing drug distribution is protein binding (Obach et al., 1997). Other approaches assume species similarity in unbound volumes of distribution or binding to tissue components (Oie and Tozer,

https://doi.org/10.1124/dmd.118.083642.

SThis article has supplemental material available at dmd.aspetjournals.org.
1979; Obach et al., 1997; McGinnity et al., 2007; Hosea et al., 2009; Berry et al., 2011). Volumes of distribution have been predicted according to the tissue composition equations proposed by Poulin and Theil (2000, 2002) and Poulin et al. (2001), as corrected by Berezhkovskiy (2004) and equations proposed by Rodgers et al. (2005) and Rodgers and Rowland (2006, 2007). Both methods calculate tissue-to-plasma concentration ratio $\left(\mathrm{P}_{\mathrm{t}: \mathrm{p}}\right)$ value as a function of tissue versus plasma values based on the sum of partitioning and binding into individual tissue components (e.g., neutral lipids, phospholipids, macromolecular proteins, and water). Partitioning of a drug into these tissue components is assumed to be driven by the lipophilicity of the drug. Both methods use the octanol/water partition coefficients or the vegetable oil/water partition coefficients as surrogates for partitioning into neutral lipids. The binding of drugs to specific macromolecular proteins is reportedly common in plasma and interstitial tissue spaces and was considered. The method proposed by Rodgers et al. (2005) and Rodgers and Rowland (2006, 2007) also considers the ionization states of drugs.

The effectiveness of these approaches for predicting distribution volumes has been assessed only on the distribution volume at steady state $\left(V_{\mathrm{ss}}\right)$. Predictions of $V_{\mathrm{ss}}$ alone are not sufficient to predict plasma concentration profiles because many drugs exhibit multiphasic eliminations

ABBREVIATIONS: AAFE, absolute average fold error; BCS, Biopharmaceutics Classification System; $C L_{\mathrm{iv}}$, plasma clearance after intravenous administration; $f_{\mathrm{u}, \mathrm{p}}$, fraction unbound in plasma; $f_{\mathrm{u}, \mathrm{t}}$, fraction unbound in human tissue; $\log P$, log of partition coefficient; PBPK, physiologically based pharmacokinetic; $P_{t: p}$, tissue-to-plasma concentration ratio; ResSS, residual sum of squares corrected with the number of observed time points; $t_{1 / 2}$, half-life; $V_{\beta}$, volume of distribution at elimination phase; $V_{1}$, volume of distribution just after intravenous administration; $V_{\mathrm{ss}}$, volume of distribution at steady state; $V_{\text {unbound }}$, unbound distribution volumes. 
(e.g., two-phase elimination). Accurate predictions of the distribution volume in the terminal elimination phase $\left(V_{\beta}\right)$ are essential for the accurate prediction of elimination $t_{1 / 2}$ values and therefore provide beneficial information for planning first-in-human clinical studies.

Physiologically based pharmacokinetic (PBPK) models have been developed to assemble individual processes in complex biologic systems into one global model (Bischoff, 1986) and to map drug transport schemes onto a physiologically realistic compartmental structure, including physiologic information and processes such as tissue composition and blood flow along with drug-specific biochemical parameters, intrinsic clearance, and $\mathrm{P}_{\mathrm{t}: \mathrm{p}}$ for each model compartment. Mechanistic tissue partition equations are built into PBPK models that incorporate $V_{\text {ss. }}$. In the process of simulating plasma concentration profiles, PBPK models evaluate $V_{\mathrm{ss}}$ and indirectly consider other distribution volumes in various elimination phases. In the current study, the accuracy of these distribution volumes predicted using PBPK models was assessed and compared with the values calculated from other proposed methods, which have been assessed in $V_{\mathrm{ss}}$ only.

It is difficult to establish the relative utility of these mechanistic tissue partition equations for predicting distribution volumes because one method cannot predict the distribution volumes of all compounds accurately (De Buck et al., 2007; Allan et al., 2008; Poulin and Theil, 2009; Rowland et al., 2011). Berry et al. (2011) and Jones et al. (2011b) reported that $V_{\mathrm{ss}}$ values predicted by one mechanistic equation were less accurate than those predicted using the unbound $V_{\mathrm{ss}}$ or fraction unbound in human tissue $\left(f_{\mathrm{u}, \mathrm{t}}\right)$ equivalency approaches. However, the effectiveness of selecting an appropriate mechanistic method for predicting distribution volumes for each compound in PBPK models has not been evaluated.

PBPK models are applied extensively in the pharmaceutical industry. In addition to the simulation of drug-drug interactions, one of the common purposes of PBPK modeling, the guidelines for first-in-human studies published by the European Medicines Agency (http://www.ema. europa.eu/docs/en_GB/document_library/Scientific_guideline/2017/ 07/WC500232186.pdf) mention PBPK modeling as a method for calculating the estimated exposure levels and doses in humans. Simcyp (Simcyp Limited, Sheffield, UK) is one of the most commonly used commercial PBPK modeling packages (Jamei et al., 2009). However, there are no reports of its use, in association with the development of animal PBPK models, to predict human distribution volumes prior to clinical studies. We report herein that human PBPK models based on animal PBPK modeling can effectively predict distribution volumes for various elimination phases in humans.

\section{Materials and Methods}

\section{Compound Selection and Pharmacokinetic Properties}

Tamsulosin, doxazosin, and diltiazem were purchased from Sigma-Aldrich (St. Louis, MO), and diazepam and midazolam were purchased from Fujifilm Wako Pure Chemical (Osaka, Japan). Reboxetine, oxprenolol, and raloxifene were obtained from Tocris Bioscience (Ellisville, MI), and bosentan and telmisartan were obtained from Mitsubishi Tanabe Pharma (Saitama, Japan). These 10 test compounds were selected largely on the basis of the availability of clinical intravenous data and the fact that their plasma concentration profiles exhibit two-phase elimination. An additional factor was good membrane permeability to ensure that tissue distribution was dominated by passive diffusion. Physicochemical properties, $\log$ of partition coefficient $(\log P)$ and $\mathrm{p} K_{\mathrm{a}}$, were determined in silico by using ACD/Percepta (version 14; Advanced Chemistry Development, Toronto, ON, Canada). In vitro plasma protein binding ratios (in rats, monkeys, dogs, and humans) and membrane permeability were determined using triplicate equilibrium dialysis and a cultured Caco-2 cell monolayer system. The Biopharmaceutics Classification System (BCS) class was determined based on the demands of the properties of compounds found in the literature $(\mathrm{Wu}$ and Benet, 2005; Gu et al., 2007; Bandela and Anupama, 2009; Tannergren et al., 2009; Benet et al., 2011; Erceg et al., 2012; Rao et al., 2012).
In vivo plasma concentration-time profiles of the 10 test compounds were measured in rats, monkeys, and dogs. The following animals received bolus intravenous administration of each compound: five male Sprague-Dawley rats (200-279 g; Charles River Japan, Kanagawa, Japan); four male cynomolgus monkeys (2.24-3.95 kg; Eve Bio-Science, Wakayama, Japan); and four male beagle dogs (8.88-12.2 kg; Oriental Yeast, Tokyo, Japan). All animal experiments were approved by the Experimental Animal Care and Use Committee of the Mitsubishi Tanabe Pharma Corporation (Saitama, Japan) and were conducted in accordance with the Declaration of Helsinki and the guidelines of the ethics committee. Each compound was detected using a liquid chromatography-tandem mass spectrometry system in either positive or negative ion mode after tuning the mass spectrometer to obtain the highest-intensity mass transition. Reported plasma concentration-time profiles after intravenous administration in humans were taken from the literature (Klotz et al., 1976; Mason and Winer, 1976; Kölle et al., 1983; Vincent et al., 1983; Weber et al., 1996; Food and Drug Administration, 1997, 1998; van Hoogdalem et al., 1997; Fleishaker et al., 1999; Palkama et al., 1999). The plasma clearance after intravenous administration $\left(C L_{\mathrm{iv}}\right)$, and the volume of distribution just after intravenous administration $\left(V_{1}\right), V_{\mathrm{ss}}$, and $V_{\beta}$ were calculated by two-compartment model analyses using WinNonlin (version 6.3; Pharsight, St Louis, MO) based on plasma concentration-time profiles experimentally obtained in animals in this study or digitally extracted from the literature for humans. For consistency with PBPK modeling analyses, if no body weight was reported for humans in literature sources, the body weight was assumed to be $80.706 \mathrm{~kg}$, a representative value for a healthy subject population implemented in the PBPK modeling package, the Simcyp simulator. The pharmacokinetic parameters obtained in silico, in vitro, or in vivo for each compound are summarized in Table 1. Plasma clearance after intravenous administration of the 10 test compounds in this study was considered to be dependent on hepatic clearance: the levels of urinary excretion of these compounds were confirmed to be low $(<10 \%)$ according to a report (Benet et al., 2011) and a textbook (Goodman and Gilman, 2005).

\section{Prediction of Distribution Volumes Using Conventional Approaches}

To facilitate comparisons with the distribution volumes predicted by PBPK modeling, several conventional approaches were used to predict human distribution volumes based on those in rats, monkeys, and dogs. Each approach was applied to the prediction of three volumes of distribution: $V_{1}, V_{\mathrm{ss}}$, and $V_{\beta}$, assuming a two-compartment model.

Approach 1-Allometric Scaling. The volumes of distribution of drugs obtained in preclinical animal species were plotted versus body weights using a log$\log$ scale. The following allometric power function (eq. 1) was then fitted to the data:

$$
V=a W^{b}
$$

where $V$ is the volume of distribution in liters, $W$ is the body weight in kilograms, and $a$ and $b$ are the allometric coefficient and exponent, respectively. The volume of distribution in humans was extrapolated using the fitted function for each drug. The body weights for rats, monkeys, and dogs were the actual body weights in in vivo pharmacokinetic analyses; that for humans was set at $70 \mathrm{~kg}$.

Approach 2-Allometric Scaling Based on the Unbound Distribution Volume. Allometric scaling based on the unbound distribution volume ( $\left.V_{\text {unbound }}\right)$ uses the same principle as allometric scaling, only substituting $V_{\text {unbound }}$, which is $V$ divided by the plasma unbound fraction.

Approach 3-The $V_{\text {unbound }}$ Equivalency Approach. The $V_{\text {unbound }}$ values of drugs in humans were assumed the same as the $V_{\text {unbound }}$ values in animals. The $V_{\text {unbound }}$ equivalency approach was conducted using the mean $V_{\text {unbound }}$ values in liters per kilogram body weight across all three animal species.

Approach 4-The $f_{\mathrm{u}, \mathrm{t}}$ Equivalency Approach. The $f_{\mathrm{u}, \mathrm{t}}$ values were assumed to be the same as those in animals. The following equation (eq. 2), using observed $V$ and fraction unbound in plasma $\left(f_{\mathrm{u}, \mathrm{p}}\right)$ values, was used for calculating $f_{\mathrm{u}, \mathrm{t}}$ :

$$
f_{u, t}=V_{t} \times f_{u, p} /\left(V-V_{p}\right)
$$

where $V_{\mathrm{p}}$ is the total volume of plasma in the animal and $V_{\mathrm{t}}$ is animal volume excluding plasma (which is essentially $1-V_{\mathrm{p}}$ (liter/kilogram), assuming that $1 \mathrm{~kg}$ of body weight equals 11 of volume. The $V_{\mathrm{p}}$ values in rats, monkeys, dogs, and humans used in the calculations were $0.0313,0.0515,0.0448$, and $0.0436 \mathrm{l} / \mathrm{kg}$, respectively.

Predicted distribution volumes, $V_{1}, V_{\mathrm{ss}}$, and $V_{\beta}$ from each approach, were used to calculate plasma concentration $\left(C_{\mathrm{p}}\right)$-time $(t)$ profiles after intravenous 
administrations of the 10 test compounds using two-compartment models with observed/reported $C L_{\mathrm{iv}}$ in humans as described in the following equation (eq. 3).

$$
\begin{aligned}
C_{\mathrm{p}}= & \frac{D}{V_{1} \cdot C L_{\mathrm{iv}}(\beta-\alpha)}\left\{\left(V_{1} \cdot \alpha \beta-\alpha \cdot C L_{\mathrm{iv}}\right) \cdot e^{-\alpha t}\right. \\
& \left.+\left(\beta \cdot C L_{\mathrm{iv}}-V_{1} \cdot \alpha \beta\right) \cdot e^{-\beta t}\right\}
\end{aligned}
$$

where

$$
\alpha=\frac{C L_{\mathrm{iv}}\left(V_{\beta}-V_{1}\right)}{V_{1}\left(V_{\beta}-V_{\mathrm{ss}}\right)}, \beta=\frac{C L_{\mathrm{iv}}}{V_{\beta}}
$$

and $D$ is the dose.

$t_{1 / 2}$ values at terminal phase were calculated with predicted $V_{\beta}$ and observed/ reported in vivo $C L_{\mathrm{iv}}$ values as follows (eq. 4):

$$
t_{1 / 2}=\frac{\ln 2}{C L_{\mathrm{iv}} / V_{\beta}}
$$

\section{PBPK Modeling for Predicting Distribution Volumes}

The Simcyp simulator (version 16) was used to perform PBPK modeling. The volumes of distribution predicted by Simcyp were derived from tissue composition-based equations that calculated the $\mathrm{P}_{\mathrm{t}: \mathrm{p}}$ values in different organs. Two published mechanistic methods are available in Simcyp for predicting $\mathrm{P}_{\mathrm{t}: \mathrm{p}}$ values based on $\log P$ and in vitro $f_{\text {u,p }}$ parameters. Method 1 is based on the approach of Poulin and Theil (2000) and Poulin et al. (2001) with the correction by Berezhkovskiy (2004), and method 2 is based on the approach of Rodgers et al. (2005) and Rodgers and Rowland (2006). $V_{\mathrm{ss}}$ was then calculated by incorporating the $\mathrm{P}_{\text {t:p }}$ values determined for each tissue. The distribution volumes of the central compartment and that for the elimination phase ( $V_{1}$ and $V_{\beta}$ in two-compartment models) were calculated indirectly in the process of simulating plasma concentration-time profiles in Simcyp with various factors, such as $P_{t: p}$ values, the cellular membrane permeabilities of drugs, blood flow rates, and the surface areas of tissues. The distribution volumes $\left(V_{1}, V_{\mathrm{ss}}\right.$, and $\left.V_{\beta}\right)$ in humans were extracted from the plasma concentration-time profiles in humans simulated in Simcyp using twocompartment model analyses with WinNonlin.

Human volumes of distribution were predicted by taking into account the results of animal PBPK models. The partitioning of drugs into individual tissue components, such as neutral lipids, phospholipids, and tissue water, was assumed to be driven by the lipophilicity of the drugs (i.e., it could be expressed as a $\log P$ value). $\log P$ values were optimized as the estimation of lipophilicity in the model for describing the distribution volumes, and the same optimized values were used for all three preclinical animals. Briefly, PBPK models of the 10 test compounds in rats, monkeys, and dogs were developed by the optimization of $\log P$ and selection of the method for $\mathrm{P}_{\mathrm{t}: \mathrm{p}}$ prediction based on the accuracy of the fit to the plasma concentration-time profiles for intravenous administration. Other parameters were not changed from the original values adopted in the Simcyp model and the measured pharmacokinetic parameters. The observed plasma clearance values after intravenous administration were used as the in vivo clearance in the PBPK models. To predict human volumes of distribution, human PBPK models were developed using the same optimized $\log P$ value and the same method for $\mathrm{P}_{\mathrm{t}: \mathrm{p}}$ prediction. These $\log P$ values and $\mathrm{P}_{\mathrm{t}: \mathrm{p}}$ prediction methods were found to provide a good fit between the simulated plasma concentration-time profiles in animal PBPK models and the observed ones for all three preclinical animals. The human pharmacokinetic parameters, such as $f_{\mathrm{u}, \mathrm{p}}$ and in vivo clearance, used in the human PBPK models were those obtained from in vitro experiments and published reports, respectively. The fixed blood/plasma ratio of 1 was used for PBPK modeling because estimated blood/plasma ratios in humans were calculated to be almost 1 in all compounds from the experimentally obtained values in rats. A representative for a healthy subject population (described as a Sim-Healthy Volunteer population) was in used Simcyp simulations with the dose regimen matched to those of the corresponding clinical pharmacokinetic studies.

In addition, the predicted distribution volumes derived from the human PBPK models with the $\log P$ value and subsequent tissue-to-plasma partition coefficients 
optimized in accordance with the development of animal PBPK models (method A) were compared with the distribution volumes predicted by using physicochemical properties without any adjustment from preclinical animal pharmacokinetics or without any change in parameters (methods B and C). Method B was that developed by Poulin and Theil (2000) and Poulin et al. (2001) with the correction by Berezhkovskiy (2004) and did not use any optimization. Method C was that developed by Rodgers et al. (2005) and Rodgers and Rowland (2006) and also did not use any optimization. For basic compounds, the distribution volumes predicted using the method based on the studies by Rodgers et al. (2005) and Rodgers and Rowland (2006) (method 2 in Simcyp) are recommended because this approach generally provides better prediction accuracy for $V_{\mathrm{ss}}$. However, consensus has not been achieved because conflicting arguments exist (De Buck et al., 2007; Allan et al., 2008; Poulin and Theil, 2009; Rowland et al., 2011). Since these studies assessed only $V_{\mathrm{ss}}$ or $\mathrm{P}_{\mathrm{t}: \mathrm{p}}$ and did not provide the consent-preferred method, the accuracy of predictions of $V_{1}, V_{\mathrm{ss}}$, and $V_{\beta}$ using both of the two methods without optimization was also assessed. In this comparison, the prediction accuracy of distribution volumes was assessed using the prediction accuracy of the resulting plasma concentration-time profiles. This was done because distribution volumes (along with measured clearance values as the input parameters for in vivo drug clearance in PBPK models) are reflected in the plasma concentration-time profiles.

\section{Prediction Accuracy}

For each approach and method, the predicted parameters and profiles were compared with the observed data. The percentages of compounds with a predicted $V_{1}, V_{\mathrm{ss}}, V_{\beta}$, and terminal $t_{1 / 2}$ values within 2-fold and 3-fold of the observed values were calculated. In addition, the absolute average fold error (AAFE) was calculated as shown in eq. 5 :

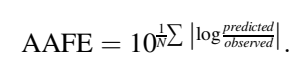

The goodness of fit of the predicted plasma concentration-time profiles to the observed plasma concentration-time profiles was assessed using the residual sum of squares corrected with the number of observed time points (ResSS). ResSS was calculated using eq. 6, where $\mathrm{Obs}_{i}$ and $\mathrm{Pred}_{i}$ represent the observed and predicted concentrations at the $i$ th time point, respectively:

$$
\operatorname{ResSS}=\sum_{i} \frac{1}{n}\left[\log \left(\operatorname{Obs}_{i}\right)-\log \left(\operatorname{Pred}_{i}\right)\right]^{2}
$$

\section{Results}

Distribution Volumes of Drugs Estimated Using Conventional Approaches and PBPK Modeling. The 10 test compounds selected in this study have a wide range of physicochemical and pharmacokinetic properties, except that they are hydrophobic (calculated in silico, $\log P,>2$ ) and minimally excreted unchanged in the kidneys (urinary excretion, $<10 \%)$. The prediction accuracy of $V_{1}, V_{\mathrm{ss}}$, and $V_{\beta}$ was assessed for the 10 compounds. The observed and predicted values for $V_{1}, V_{\mathrm{ss}}$, and $V_{\beta}$ using conventional approaches 1-4 (allometric scaling, allometric scaling based on the unbound distribution volume, the $V_{\text {unbound }}$ equivalency approach, and the $f_{\mathrm{u}, \mathrm{t}}$ equivalency approach, respectively) and PBPK modeling are shown in Fig. 1. The distribution volumes predicted using each of the four conventional approaches and PBPK modeling are summarized in Table 2 . The human $V_{1}, V_{\mathrm{ss}}$, and $V_{\beta}$ values predicted using all four conventional approaches resulted in higher AAFEs $\left(3.25-3.52,1.78-2.53\right.$, and $3.12-4.46$ for $V_{1}, V_{\mathrm{ss}}$, and $V_{\beta}$, respectively) and lower percentages of the predicted values falling within 2-fold errors of the observed values (30\%-50\%, 40\%-70\%, and 30\%$40 \%$ for $V_{1}, V_{\mathrm{ss}}$, and $V_{\beta}$, respectively) (Fig. 1 , A-D; Table 2). In terms of $V_{\text {ss }}$ prediction, conventional approaches 3 and 4 (the $V_{\text {unbound }}$ equivalency and $f_{\mathrm{u}, \mathrm{t}}$ equivalency approaches) resulted in good predictions, with average fold errors of 1.78 and 1.79 , respectively. However, in terms of $V_{1}$ and $V_{\beta}$ prediction, large discrepancies (e.g., 10-fold discrepancies) between predicted and observed values were evident using conventional approaches on some compounds, leading to greater than 3-fold average errors for each conventional approach.

In contrast, the prediction of human $V_{1}, V_{\mathrm{ss}}$, and $V_{\beta}$ using PBPK modeling (Fig. 1E) resulted in $50 \%, 80 \%$, and $60 \%$ of predictions within 2-fold of the observed values, with average fold errors of 2.14, 1.72, and 1.67, respectively, suggesting that PBPK modeling produced the most accurate predictions. The distribution volumes predicted by PBPK modeling avoided the extremely inaccurate predictions of the conventional methods, with almost all compounds within 3-fold of the observed values; the exceptions were $V_{1}$ for raloxifene and diltiazem, and $V_{\mathrm{ss}}$ for tamsulosin.

Predicted $t_{1 / 2}$ Values and Plasma Concentration-Time Profiles after Virtual Intravenous Administration Using Distribution Volumes Predicted by Conventional Approaches and PBPK Modeling. Predicted $t_{1 / 2}$ values at the terminal phase are summarized in Table 3. Predictions of $t_{1 / 2}$ at the terminal phase using conventional approaches 1-4 showed $40 \%-50 \%$ of predictions within 3 -fold of the observed values with average fold errors of 4.46, 3.73, 3.12, and 3.11, respectively. Large discrepancies (e.g., 10-fold discrepancies) between predicted and observed values of terminal $t_{1 / 2}$ existed using conventional approaches on some compounds as observed in $V_{\beta}$ predictions. Values of the terminal $t_{1 / 2}$ for all compounds tested were within 3-fold errors with AAFEs of 1.67 using PBPK modeling methods.

The simulated human plasma concentration-time profiles after intravenous administrations were compared using the distribution volumes obtained using conventional approach 1, thought to be the most commonly used approach, and PBPK models for the 10 test compounds (Fig. 2). Also shown in Fig. 2 are the reported plasma concentrations and the curves fitted using two-compartment models. The residual sums of squares of plasma concentration-time profiles simulated based on distribution volumes obtained by PBPK modeling were smaller than those based on conventional approach 1 for 7 of 10 compounds (Supplemental Table 1). Moreover, the residual sums of squares of the plasma concentration-time profiles obtained using distribution volumes from PBPK modeling were smaller on average, indicating that the PBPK modeling methodology provided better predictions matched to the observed data (Supplemental Table 1).

Human PBPK Modeling Using Tissue-To-Plasma Partition Coefficients and Optimized Parameter Based on Insights Gained from Animal PBPK Modeling. For the 10 test compounds, the simulated human plasma concentration-time profiles after virtual intravenous administrations calculated using PBPK models were compared for distribution volumes obtained using three different prediction methods for tissue-to-plasma partition coefficients (methods A, B, and C) (Fig. 3). The residual sums of squares for the plasma concentrationtime profiles of the 10 compounds obtained using three methods are summarized in Table 4. Based on the average residual sum of squares, method A achieved the most accurate predictions for 10 compounds tested in this study, closely followed by method B. The best prediction method as assessed using the residual sum of squares differed among the compounds tested. Method A provided the most accurate prediction for reboxetine, doxazosin, bosentan, and diltiazem, whereas method B was best for tamsulosin, oxprenolol, midazolam, and raloxifene, and method $\mathrm{C}$ was best for diazepam and telmisartan.

In method $\mathrm{A}$, the optimal mechanistic method to predict $\mathrm{P}_{\text {t:p }}$ was selected from the two mechanistic methods, based on the results of animal PBPK models. As a result, the method developed in studies by Rodgers et al. (2005) and Rodgers and Rowland (2006) was selected for diazepam and telmisartan, and the method developed in the studies by Poulin and Theil (2000) and Poulin et al. (2001) with the correction by Berezhkovskiy (2004) was selected for the remaining eight compounds (Table 5). 
A Approach 1 (allometric scaling)
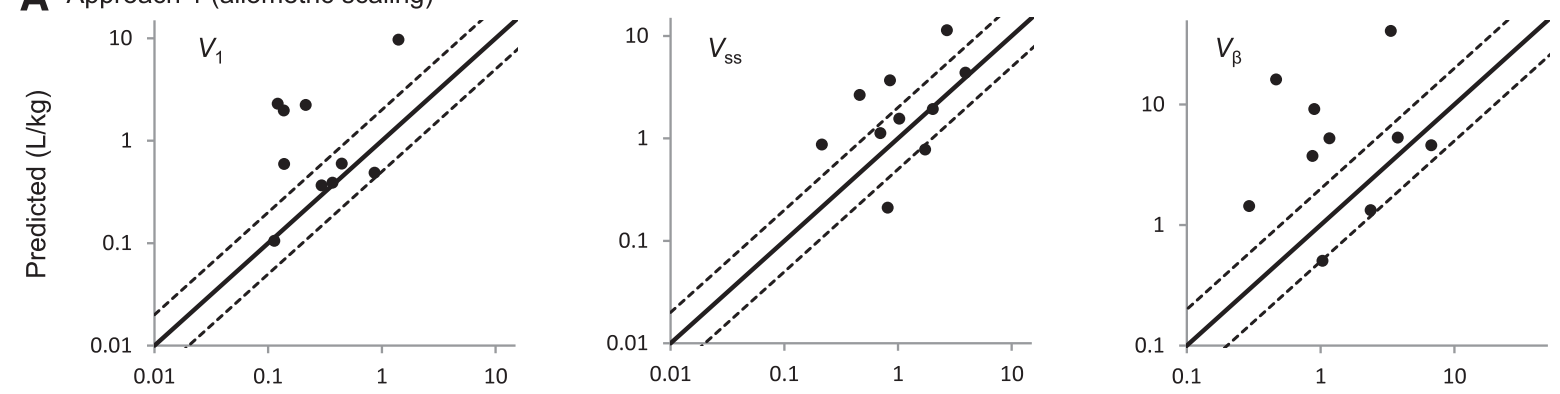

B Approach 2 (allometric scaling based on the unbound distribution volume)
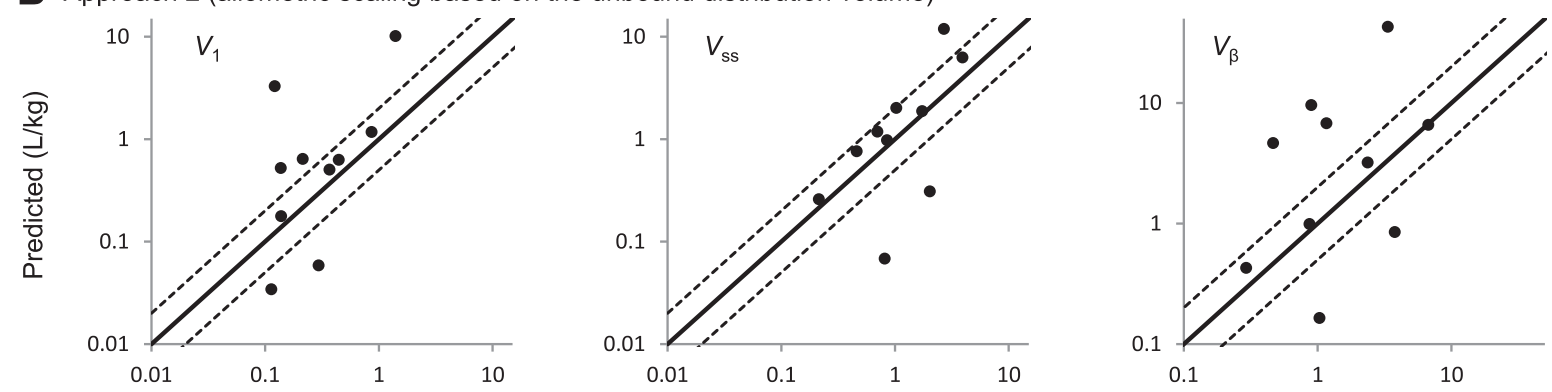

C Approach 3 (the $V_{\text {unbound }}$ equivalency approach)
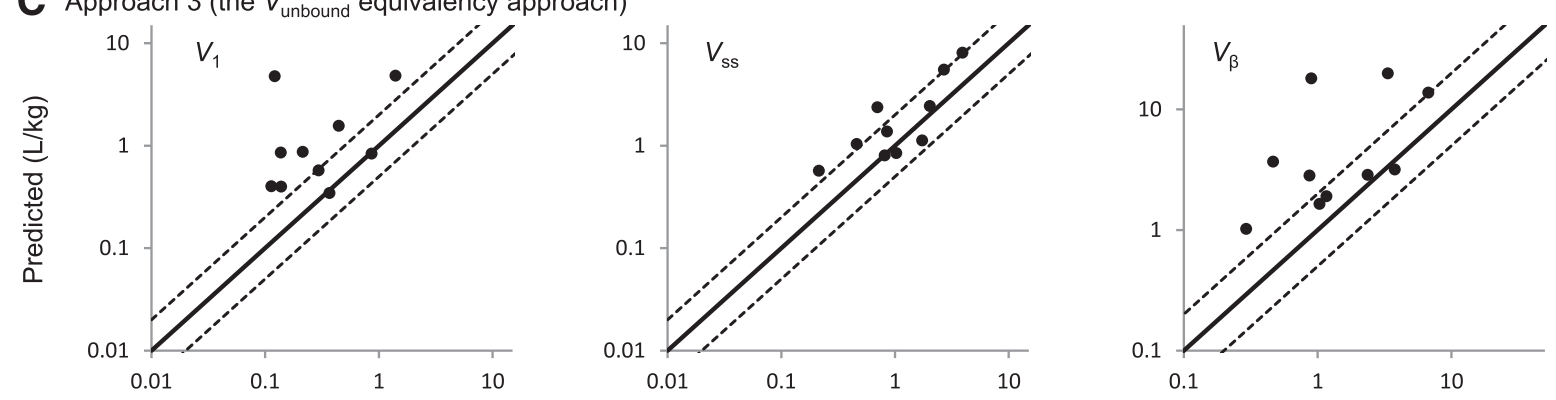

D Approach 4 (the $f_{u, t}$ equivalency approach)
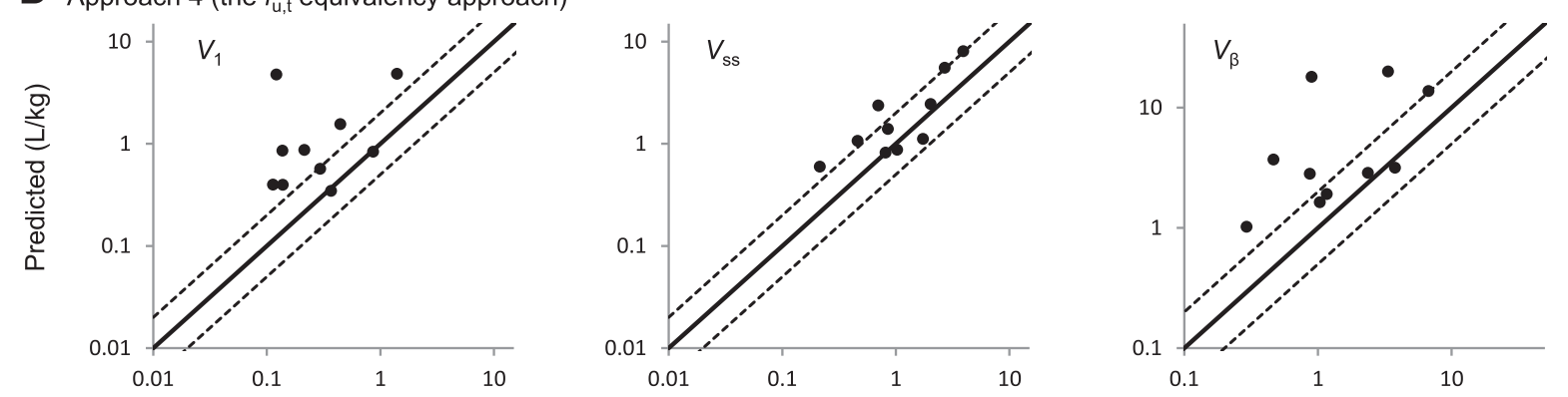

E PBPK modeling
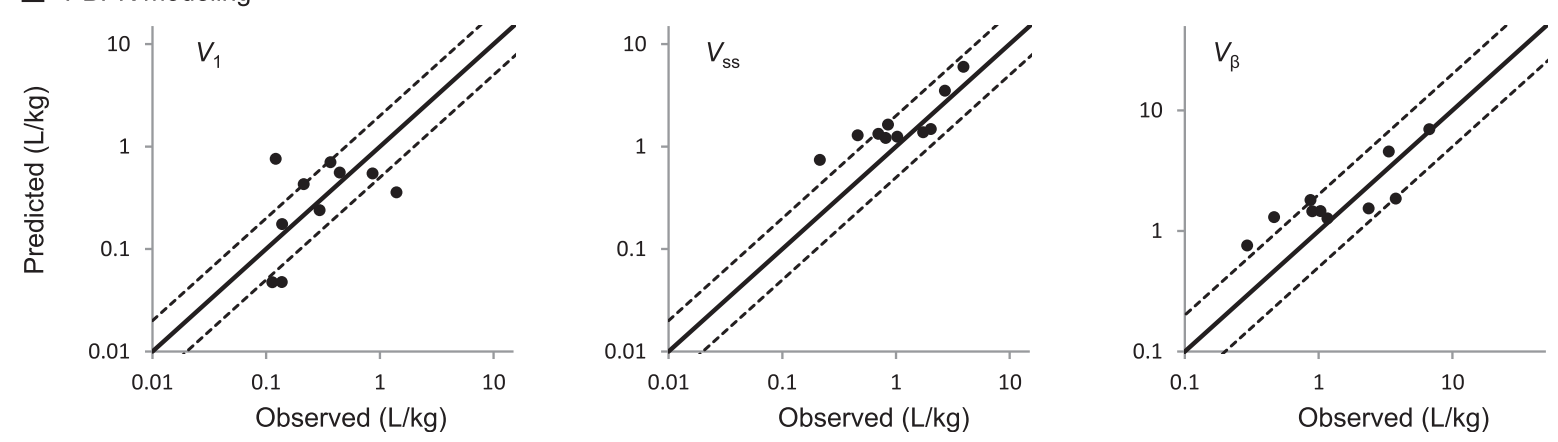

Fig. 1. Observed and predicted volumes of distribution $\left(V_{1}, V_{\mathrm{ss}}\right.$, and $\left.V_{\beta}\right)$ of 10 compounds in humans. Observed and predicted volumes of distribution were calculated by two-compartment models using approach 1 (allometric scaling) (A), approach 2 (allometric scaling based on the unbound distribution volume) (B), approach 3 (the $V_{\text {unbound }}$ equivalency approach) (C), approach 4 (the $f_{\mathrm{u}, \mathrm{t}}$ equivalency approach) (D), and PBPK modeling (E). Solid and broken lines represent no error and 2-fold error, respectively. 


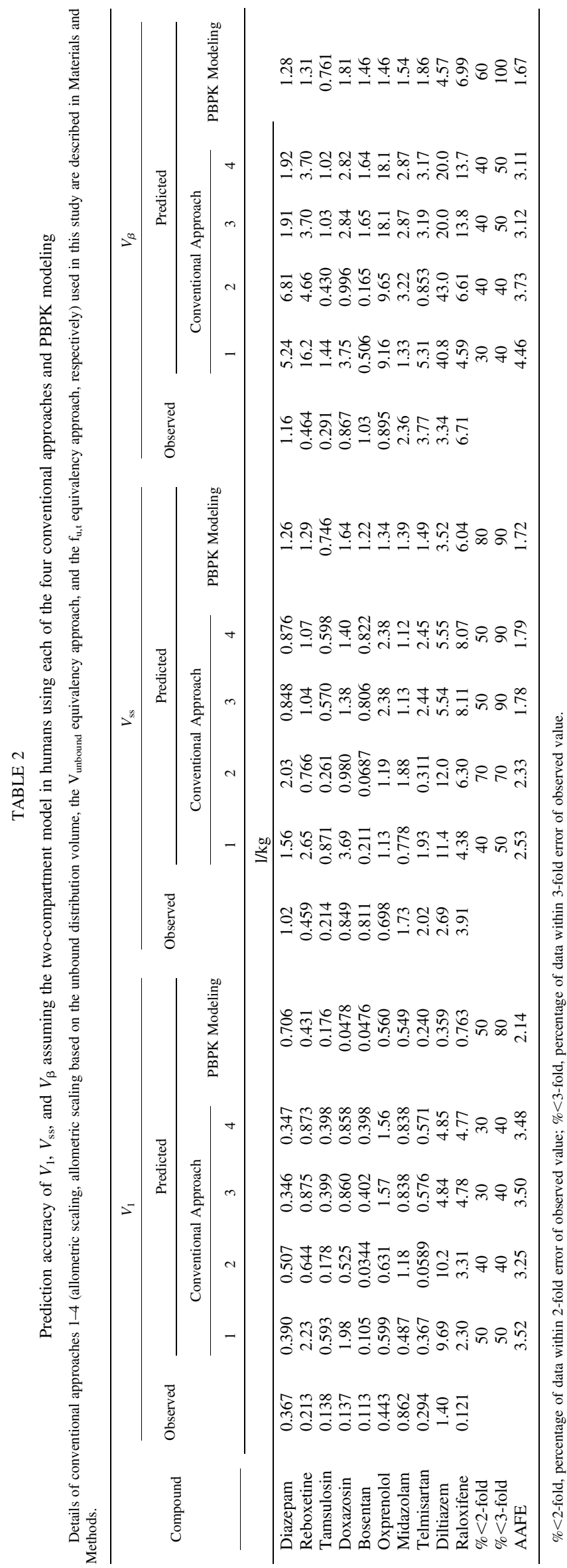

TABLE 3

Prediction accuracy of terminal $t_{1 / 2}$ values in humans after virtual intravenous administrations modeled based on distribution volumes obtained using approaches $1-4$ and PBPK modeling

\begin{tabular}{|c|c|c|c|c|c|c|}
\hline \multirow{4}{*}{ Compound } & \multicolumn{6}{|c|}{$t_{1 / 2}(\mathrm{~h})$} \\
\hline & \multirow{3}{*}{ Observed } & \multicolumn{5}{|c|}{ Predicted } \\
\hline & & \multicolumn{4}{|c|}{ Conventional Approach } & \multirow{2}{*}{ PBPK Modeling } \\
\hline & & 1 & 2 & 3 & 4 & \\
\hline & \multicolumn{6}{|c|}{$\mathrm{h}$} \\
\hline Diazepam & 38.4 & 173.1 & 224.9 & 63.0 & 63.3 & 42.2 \\
\hline Reboxetine & 8.9 & 311.0 & 89.8 & 71.2 & 71.3 & 25.1 \\
\hline Tamsulosin & 4.9 & 24.3 & 7.3 & 17.3 & 17.3 & 12.9 \\
\hline Doxazosin & 8.0 & 34.6 & 9.2 & 26.2 & 26.1 & 16.8 \\
\hline Bosentan & 4.6 & 2.3 & 0.7 & 7.4 & 7.4 & 6.6 \\
\hline Oxprenolol & 1.8 & 18.7 & 19.7 & 37.0 & 36.9 & 3.0 \\
\hline Midazolam & 3.5 & 2.0 & 4.8 & 4.3 & 4.3 & 2.3 \\
\hline Telmisartan & 5.2 & 7.3 & 1.2 & 4.4 & 4.4 & 2.6 \\
\hline Diltiazem & 3.7 & 44.8 & 47.1 & 21.9 & 21.9 & 5.0 \\
\hline Raloxifene & 7.2 & 4.9 & 7.1 & 14.8 & 14.7 & 7.5 \\
\hline$\%<2$-fold & & 30 & 40 & 40 & 40 & 60 \\
\hline$\%<3$-fold & & 40 & 40 & 50 & 50 & 100 \\
\hline AAFE & & 4.46 & 3.73 & 3.12 & 3.11 & 1.67 \\
\hline
\end{tabular}

$\%<2$-fold, percentage of data within 2 -fold error of observed value; $\%<3$-fold, percentage of data within 3-fold error of observed value. Conventional approaches $1-4$ represent allometric scaling, allometric scaling based on the unbound distribution volume, the $V_{\text {unbound }}$ equivalency approach, and the $f_{\mathrm{u}, \mathrm{t}}$ equivalency approach, respectively.

\section{Discussion}

One aim of the present study was to evaluate the prediction accuracy of distribution volumes for various phases estimated using PBPK modeling in humans developed in accordance with animal PBPK models by comparison with those obtained using conventional approaches. Another aim was to evaluate the effectiveness of selecting an appropriate mechanistic method to determine the $\mathrm{P}_{\text {t:p }}$ values used for predicting distribution volumes during PBPK model development based on animal pharmacokinetic models.

The effectiveness of various approaches for predicting $V_{\mathrm{ss}}$ has been assessed. Jones et al. (2011b) assessed the prediction accuracy of 24 prediction methods for $V_{\mathrm{ss}}$, including each mechanistic equation for $\mathrm{P}_{\text {t:p }}$. Berry et al. (2011) assessed additionally the $V_{\text {unbound }}$ equivalency for the $V_{\mathrm{ss}}$ approach among allometric scaling, the $f_{\mathrm{u}, \mathrm{t}}$ equivalency approach, and two mechanistic equations. As for $V_{\mathrm{ss}}$ prediction, the results in this study were consistent with those reports with respect to the fact that conventional approaches were well able to predict $V_{\mathrm{ss}}$, especially for approaches 3 and 4 ( $V_{\text {unbound }}$ and $f_{\mathrm{u}, \mathrm{t}}$ equivalency approaches). We also evaluated the prediction accuracy of those approaches for $V_{1}$ and $V_{\beta}$ because they are essential to predict plasma concentration profiles exhibiting two-phase elimination. In the prediction of $V_{1}$ and $V_{\beta}$, some discrepancies between predicted and observed values were seen from conventional approaches on some compounds (Fig. 1; Table 2). Most of the $V_{1}$ and $V_{\beta}$ values with more than 3 -fold errors were overpredicted. The reason why $V_{1}$ values were predicted with relatively large errors was not clear, but possible reasons might be allometric differences in plasma or tissue distribution. One of the reasons for the discrepancies in $V_{\beta}$ predicted using conventional approaches might be the species differences in clearance values. This is because the distribution volume in the elimination phase is affected by the clearance of the compound because of the apparent steady state in distribution between peripheral tissues and blood/plasma. In conventional approaches, species differences in clearances are not considered when scaling $V_{\beta}$ from animals to humans. Overprediction of $V_{\beta}$ suggested relatively small clearance values in humans compared with 

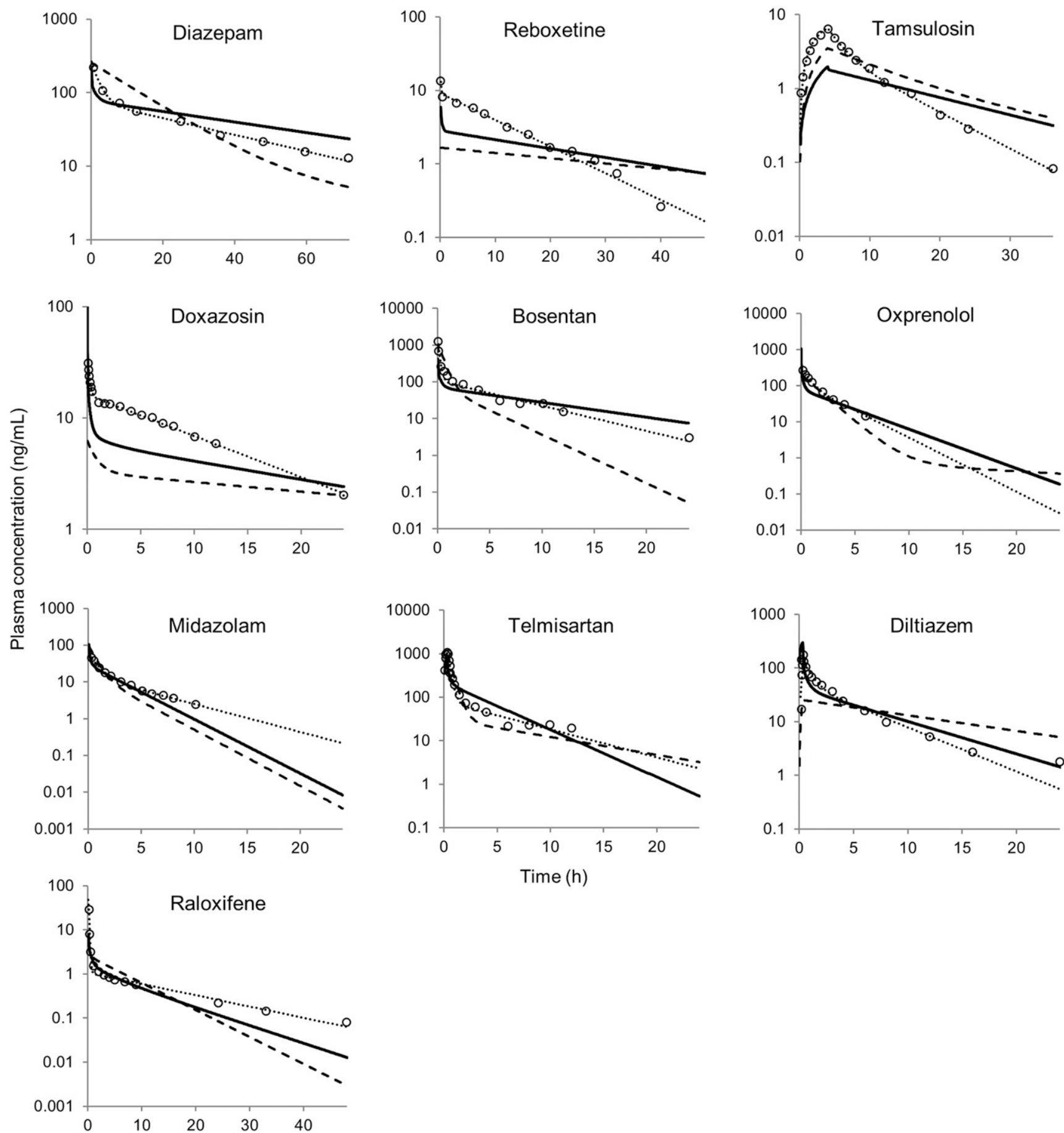

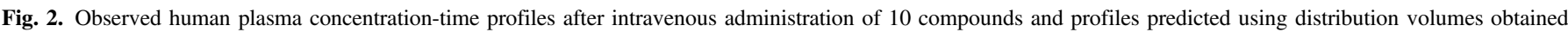

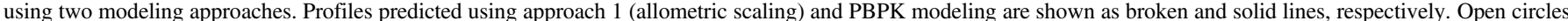

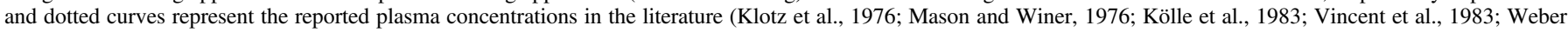

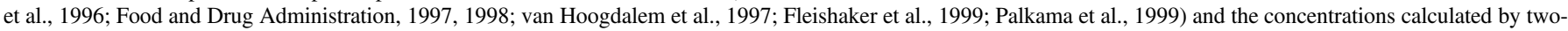
compartment models, respectively.

those in animals or scaled from animals. Other contributing factors explaining the difficulties in predicting $V_{\beta}$ values may be inappropriate estimation of $V_{\beta}$ in animals based on compartment model analyses with drug concentrations below the lower limits of quantification and limited numbers of time points at the elimination phase. These discrepancies in predicting distribution volumes indicated insufficiencies in the conventional approaches for predicting multiphasic plasma concentration profiles.
In other reports, $V_{\mathrm{ss}}$ values predicted by one mechanistic equation were shown to be less accurate compared with the $V_{\text {unbound }}$ or $f_{\mathrm{u}, \mathrm{t}}$ equivalency approaches (Berry et al., 2011; Jones et al., 2011b). We evaluated the effectiveness of modifying the lipophilicity input of compounds and selecting an appropriate mechanistic method for predicting distribution volumes in PBPK models in accordance with the insights gained from animal PBPK models. The prediction of distribution volumes using such PBPK modeling avoided the above- 
Diazepam

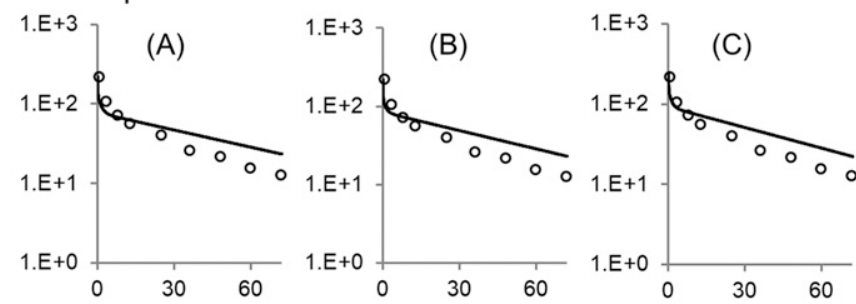

Oxprenolol
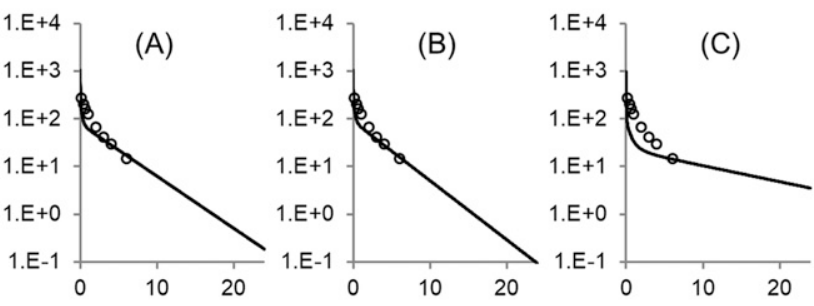

Reboxetine
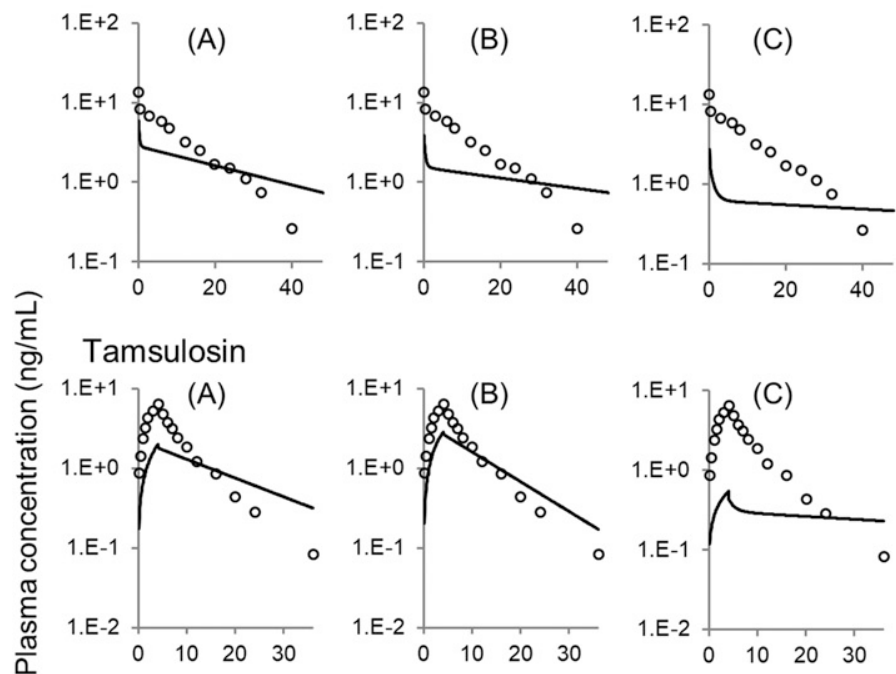

Midazolam
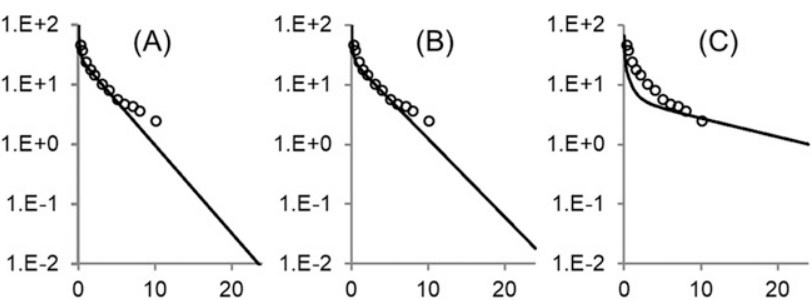

Telmisartan
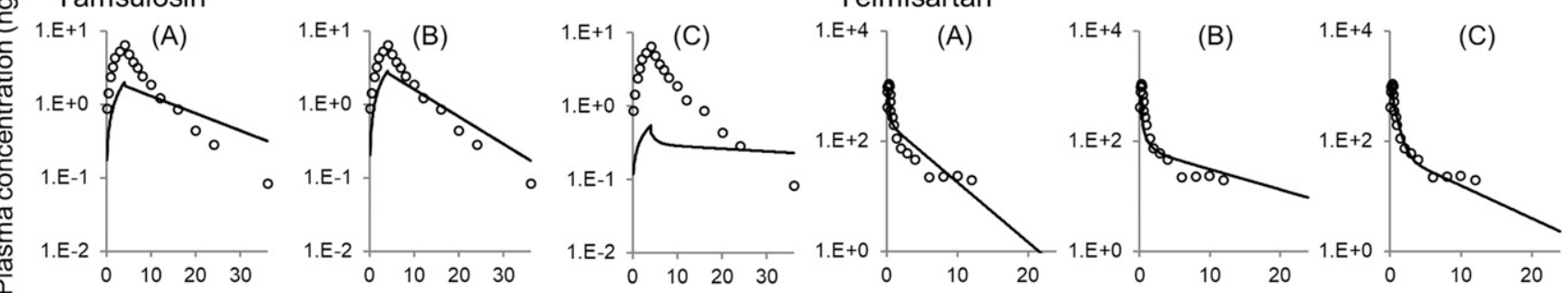

Doxazosin
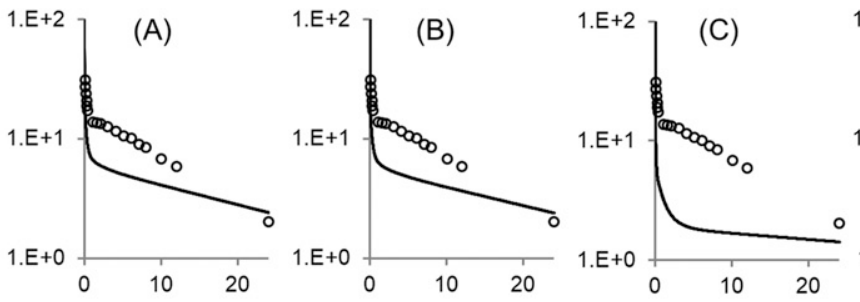

\section{Diltiazem}
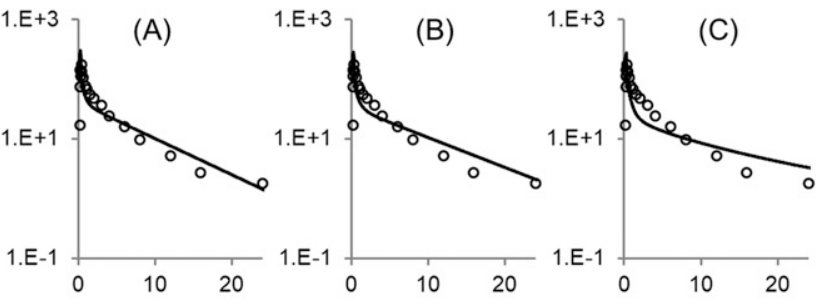

Bosentan
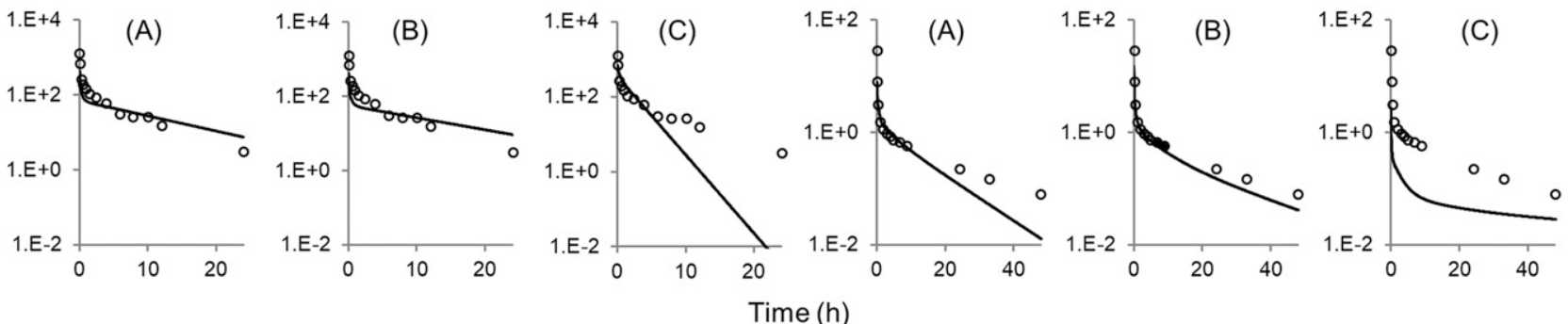

Fig. 3. Observed human plasma concentration-time profiles after intravenous administration and profiles predicted using three different prediction methods for tissue-toplasma partition coefficients. Tissue-to-plasma partition coefficients were predicted in accordance with animal PBPK models optimized with animal pharmacokinetic data, including the mechanistic method selection (method A) (A); using the mechanistic method based on the approach of Poulin and Theil (2000) and Poulin et al. (2001), with the correction by Berezhkovskiy (2004) with in silico physicochemical parameters (method B) (B); and using the mechanistic method based on the studies by Rodgers et al. (2005) and Rodgers and Rowland (2006) with in silico physicochemical parameters (method C) (C). Open circles represent the reported plasma concentrations in the literature (Klotz et al., 1976; Mason and Winer, 1976; Kölle et al., 1983; Vincent et al., 1983; Weber et al., 1996; Food and Drug Administration, 1997, 1998; van Hoogdalem et al., 1997; Fleishaker et al., 1999; Palkama et al., 1999).

mentioned inaccurate predictions of conventional methods for $V_{1}$ and $V_{\beta}$ and achieved predictions that were just as accurate as those achieved with approaches 3 and 4 ( $V_{\text {unbound }}$ and $f_{\mathrm{u}, \mathrm{t}}$ equivalency approaches) (Fig. 1; Table 2). The predicted distribution volumes obtained using the mechanistic method developed in the studies by Rodgers et al. (2005) and Rodgers and Rowland (2006) are reportedly to be preferred for basic compounds. However, their method without any optimization, method $\mathrm{C}$, did not provide the best predictions for the basic compounds tested in this study. The methods used for predicting $\mathrm{P}_{\mathrm{t} \text { : }}$ values were chosen among two mechanistic methods based on the accuracy of PBPK models 
TABLE 4

Prediction accuracy of the modeled pharmacokinetic profiles in humans after virtual intravenous administrations using three different prediction methods for tissue-toplasma partition coefficients

Method A produced values optimized with animal pharmacokinetic data and includes model selection [method developed by Poulin and Theil (2000) and Poulin et al. (2001), as corrected by Berezhkovskiy (2004); or Rodgers et al. (2005) and Rodgers and Rowland (2006)] for the prediction of tissue-to-plasma partition coefficients. Method B was that developed by Poulin and Theil (2000) and Poulin et al. (2001), as corrected by Berezhkovskiy (2004); and method C was that developed by Rodgers et al. (2005) and Rodgers and Rowland (2006) with in silico physicochemical parameters. Prediction accuracy is shown as the ResSS and the modeled plasma concentrations corrected by the number of observed time points. ResSS was calculated using the following equation: $\sum \frac{1}{n}\left[\log \left(O b s_{i}\right)-\log \left(\operatorname{Pred}_{i}\right)\right]^{2}$, where $\mathrm{Obs}_{i}$ is the observed concentration at the $i$ th time point and Pred $_{i}$ is the predicted concentration at the $i$ th time point.

\begin{tabular}{lccc}
\hline & \multicolumn{2}{c}{ Prediction Method for Tissue-to-Plasma Partition Coefficients } \\
\cline { 2 - 4 } & Method A & Method B & Method C \\
\hline Diazepam & 0.039 & 0.041 & 0.036 \\
Reboxetine & 0.091 & 0.190 & 0.452 \\
Tamsulosin & 0.180 & 0.088 & 0.746 \\
Doxazosin & 0.080 & 0.091 & 0.413 \\
Bosentan & 0.082 & 0.108 & 0.908 \\
Oxprenolol & 0.056 & 0.045 & 0.206 \\
Midazolam & 0.030 & 0.019 & 0.082 \\
Telmisartan & 0.047 & 0.093 & 0.014 \\
Diltiazem & 0.127 & 0.133 & 0.148 \\
Raloxifene & 0.132 & 0.083 & 0.867 \\
Average & 0.086 & 0.089 & 0.387 \\
\hline
\end{tabular}

of the 10 test compounds in three animal species using the same mechanistic equations and adjusted $\log P$ values (method $\mathrm{A}$ ) (Table 5). The selected mechanistic tissue partition equation in method $\mathrm{A}$ was matched to the better mechanistic equation among methods $\mathrm{B}$ and $\mathrm{C}$, model development without any adjustment of physicochemical properties. These results suggested that the choice of method for predicting $\mathrm{P}_{\text {t:p }}$ could be supported by the insights gained from animal PBPK models. It has been reported that confirming the mechanistic tissue partition equations in animal PBPK models could validate the use of mechanistic equations for $\mathrm{P}_{\mathrm{t}: \mathrm{p}}$ in human PBPK models (Jones et al., 2006, 2011a). However, it may be misleading to use animal pharmacokinetic data and PBPK models without optimization to validate mechanistic equations. This is because, for some combinations of animal species and compounds, animal PBPK models have shown discrepancies between simulated and observed concentration profiles. Those combinations with discrepancies may prevent the selection of optimal methods for $\mathrm{P}_{\mathrm{t}: \mathrm{p}}$, although method $\mathrm{B}$ without any optimization showed good prediction accuracy next to method $\mathrm{A}$ among test compounds in this study. Instead, we suggest introducing the modification of $\log P$ values and the selection of the mechanistic equation in accordance with insights gained from animal PBPK models for the development of human PBPK models.

It might be considered controversial to introduce modifications of $\log P$ as an input value for lipophilicity in PBPK modeling. However, inaccuracies in the calculation, or possibly measurement, of physicochemical properties can result in inaccuracies in predicted $\mathrm{P}_{\mathrm{t}: \mathrm{p}}$ values, as discussed by Rodgers and Rowland (2007). Partitioning into octanol or vegetable oil may not always adequately reflect the partitioning of a candidate drug into lipids in in vivo tissues. The vegetable oil/water partition coefficient is calculated from $\log P$ according to a reported regression equation (Leo et al., 1971). Therefore, it may be justified to modify $\log P$ values to obtain plasma concentration profiles matched to observed values because the physicochemical properties contain uncertainties. Modifications of $\log P$ can be verified if simulated values in animal PBPK models match the observed values in animals prior to development of the human PBPK model. In addition, modified $\log P$ could be supported unless the modified values would be unrealistic values of physicochemical considerations. Another approach to overcoming uncertainties originating from predictions based on physicochemical parameters is to introduce a scaling factor for $\mathrm{P}_{\mathrm{t}: \mathrm{p}}$ or adjustment on $f_{\mathrm{u}, \mathrm{p}}$ to make the simulated plasma concentrations or distribution volumes match the observed data. They could be helpful in the development of PBPK models to match the simulated and observed plasma concentration profiles. However, it is thought to be difficult to use them for prediction purposes because different scaling factors or adjustments on $f_{\mathrm{u}, \mathrm{p}}$ would be adopted for the three animal species because of both the overestimation and underestimation observed in animal PBPK models without any adjustment in lipophilicity, resulting in difficulties in finding an appropriate scaling factor or adjustment on $f_{\mathrm{u}, \mathrm{p}}$ in common in all three animals that can be extrapolated to humans. In addition, $f_{\mathrm{u}, \mathrm{p}}$ should be inherent in each animal species. Therefore, we suggest that modification of $\log P$, as the estimation of lipophilicity in the model, be used to describe the distribution volumes in all three preclinical animals in common. No modification of $\mathrm{p} K_{\mathrm{a}}$, another in silico parameter, due to the modification of $\log P$ and selection of an appropriate method for $\mathrm{P}_{\mathrm{t}: \mathrm{p}}$ with in silico $\mathrm{p} K_{\mathrm{a}}$ could sufficiently express simulated plasma concentration profiles matching with the observed profiles in animal PBPK models of three animal species and accurately predict distribution volumes, resulting in plasma concentration profiles in human PBPK models.

TABLE 5

Selected mechanistic method for tissue-to-plasma concentration ratio and optimized logP value in human PBPK model development in accordance with animal PBPK models (method A) In Mechanistic Method for Pt:p, method 1 is the method developed by Poulin and Theil (2000) and Poulin et al. (2001) with the correction by Berezhkovskiy (2004), and method 2 is the method developed by Rodgers et al. (2005) and Rodgers and Rowland (2006)

$\Delta \log P$ represents the magnitude of modification on $\log P$ from calculated in silico values gained from animal PBPK model development

\begin{tabular}{lccr}
\hline Compound & Mechanistic method for $\mathrm{P}_{\text {t:p }}$ & Optimized $\log P$ & $\Delta \log P$ \\
\hline Diazepam & method 2 & 3.05 & 0.08 \\
Reboxetine & method 1 & 2.84 & -0.43 \\
Tamsulosin & method 1 & 2.61 & 0.44 \\
Doxazosin & method 1 & 3.50 & -0.04 \\
Bosentan & method 1 & 4.00 & -0.17 \\
Oxprenolol & method 1 & 2.20 & 0.10 \\
Midazolam & method 1 & 3.35 & -0.08 \\
Telmisartan & method 2 & 4.30 & -3.00 \\
Diltiazem & method 1 & 3.35 & -0.30 \\
Raloxifene & method 1 & 4.80 & -2.07 \\
\hline
\end{tabular}


The 10 test compounds used in the current study were selected because they possessed high membrane permeability, which justified the assumption that the tissue distribution was dominated by passive diffusion. All 10 compounds belong to BCS class I or II. The prediction accuracy of compounds that, because of low membrane permeability, are distributed to tissues mainly by active transport should be investigated further.

PBPK modeling in humans developed in accordance with insights gained from animal PBPK models, including optimization of in silico physicochemical parameters and selection of the mechanistic equation for $\mathrm{P}_{\mathrm{t}: \mathrm{p}}$ prediction, was able to facilitate the prediction of the plasma concentration-time profiles of drugs that exhibit multiphasic elimination. This approach was able to more accurately predict values of $V_{1}, V_{\mathrm{ss}}$, and $V_{\beta}$, and will therefore be useful for predicting terminal phase $t_{1 / 2}$ values, compared with conventional approaches. This, in turn, should contribute to the improved design of clinical studies by more accurately estimating the required sampling times and duration, and the number of doses until steady state prior to first-in-human studies. Regarding some additional benefits, one is to estimate the effects of drug-drug interactions and to investigate the pharmacokinetics in special populations, such as the elderly or patients with hepatic/renal impairment, at the preclinical stage or just after first-in-human studies. Another benefit is the possibility of resolving the issue that oral absorption and bioavailability may not be well described separately from volumes of distribution and clearance values in PBPK models developed based on pharmacokinetic data from oral administrations only. Moreover, this is the first study to confirm the strategy of animal PBPK modeling prior to human PBPK modeling using Simcyp, which has recently improved its animal model lineup. The good predictions obtained from PBPK modeling were derived from the effective description of complicated drug movements, such as distribution schemes and rates of transport to tissues, estimated from the biochemical and physicochemical properties of drugs and the physiologic parameters implemented in Simcyp.

\section{Acknowledgments}

We thank Drs. Makiko Shimizu and Toshiyuki Kume for their help, and David Smallbones for editing a draft of this manuscript.

\section{Authorship Contributions}

Participated in research design: Shimizu, Nakada, and Nakamaru.

Conducted experiments: Shimizu, Kojima, Ogasawara, and Nakamaru.

Contributed new reagents or analytic tools: Shimizu, Kojima, Ogasawara, and Nakamaru.

Performed data analysis: Shimizu and Yoshida.

Wrote or contributed to the writing of the manuscript: Shimizu, Nakada, Nakamaru, and Yamazaki.

\section{References}

Allan G, Davis J, Dickins M, Gardner I, Jenkins T, Jones H, Webster R, and Westgate H (2008) Pre-clinical pharmacokinetics of UK-453,061, a novel non-nucleoside reverse transcriptase inhibitor (NNRTI), and use of in silico physiologically based prediction tools to predict the oral pharmacokinetics of UK-453,061 in man. Xenobiotica 38:620-640.

Bandela JJ and Anupama CH (2009) Advanced PEGylation for the development of raloxifene hydrochloride, BCS class II drug. J Young Pharm 1:295-300.

Benet LZ, Broccatelli F, and Oprea TI (2011) BDDCS applied to over 900 drugs. AAPS J 13 519-547.

Berezhkovskiy LM (2004) Volume of distribution at steady state for a linear pharmacokinetic system with peripheral elimination. J Pharm Sci 93:1628-1640.

Berry LM, Li C, and Zhao Z (2011) Species differences in distribution and prediction of human V (ss) from preclinical data. Drug Metab Dispos 39:2103-2116.

Bischoff KB (1986) Physiological pharmacokinetics. Bull Math Biol 48:309-322.

Boxenbaum H (1982) Interspecies scaling, allometry, physiological time, and the ground plan of pharmacokinetics. J Pharmacokinet Biopharm 10:201-227.

De Buck SS, Sinha VK, Fenu LA, Nijsen MJ, Mackie CE, and Gilissen RA (2007) Prediction of human pharmacokinetics using physiologically based modeling: a retrospective analysis of 26 clinically tested drugs. Drug Metab Dispos 35:1766-1780.

Erceg M, Vertzoni M, Cerić H, Dumić M, Cetina-Čižmek B, and Reppas C (2012) In vitro vs. canine data for assessing early exposure of doxazosin base and its mesylate salt. Eur J Pharm Biopharm 80:402-409.

Fleishaker JC, Mucci M, Pellizzoni C, and Poggesi I (1999) Absolute bioavailability of reboxetine enantiomers and effect of gender on pharmacokinetics. Biopharm Drug Dispos 20:53-57.
Food and Drug Administration (1997) New drug approval: raloxifene hydrochloride tablets. FDA approval package. Clinical Pharmacology and Biopharmaceutics Review 020815/S-000:12. Food and Drug Administration, Silver Spring, MD.

Food and Drug Administration (1998) New drug approval: telmisartan tablets. FDA approval package. Clinical Pharmacology and Biopharmaceutics Review 020850/S-000:6. Food and Drug Administration, Silver Spring, MD.

Goodman LS and Gilman A (2005) The Pharmacological Basis of Therapeutics, 11th ed, McGrawHill Publishers, New York

Gu C-H, Li H, Levons J, Lentz K, Gandhi RB, Raghavan K, and Smith RL (2007) Predicting effect of food on extent of drug absorption based on physicochemical properties. Pharm Res 24 $1118-1130$.

Hosea NA, Collard WT, Cole S, Maurer TS, Fang RX, Jones H, Kakar SM, Nakai Y, Smith BJ, Webster R, et al. (2009) Prediction of human pharmacokinetics from preclinical information: comparative accuracy of quantitative prediction approaches. J Clin Pharmacol 49:513-533.

Jamei M, Marciniak S, Feng K, Barnett A, Tucker G, and Rostami-Hodjegan A (2009) The Simcyp population-based ADME simulator. Expert Opin Drug Metab Toxicol 5:211-223.

Jones HM, Gardner IB, Collard WT, Stanley PJ, Oxley P, Hosea NA, Plowchalk D, Gernhardt S, Lin J, Dickins M, et al. (2011a) Simulation of human intravenous and oral pharmacokinetics of 21 diverse compounds using physiologically based pharmacokinetic modelling. Clin Pharmacokinet 50:331-347.

Jones HM, Parrott N, Jorga K, and Lavé T (2006) A novel strategy for physiologically based predictions of human pharmacokinetics. Clin Pharmacokinet 45:511-542.

Jones RD, Jones HM, Rowland M, Gibson CR, Yates JW, Chien JY, Ring BJ, Adkison KK, Ku MS, He H, et al. (2011b) PhRMA CPCDC initiative on predictive models of human pharmacokinetics, part 2: comparative assessment of prediction methods of human volume of distribution. J Pharm Sci 100:4074-4089.

Klotz U, Antonin KH, and Bieck PR (1976) Pharmacokinetics and plasma binding of diazepam in man, dog, rabbit, guinea pig and rat. J Pharmacol Exp Ther 199:67-73.

Kölle EU, Ochs HR, and Vollmer KO (1983) Pharmacokinetic model of diltiazem. Arzneimittelforschung 33:972-977.

Leo A, Hansch C, and Elkins D (1971) Partition coefficients and their uses. Chem Rev 71:525-616. Mason WD and Winer N (1976) Pharmacokinetics of oxprenolol in normal subjects. Clin Pharmacol Ther 20:401-412.

McGinnity DF, Collington J, Austin RP, and Riley RJ (2007) Evaluation of human pharmacokinetics, therapeutic dose and exposure predictions using marketed oral drugs. Curr Drug Metab 8: 463-479.

Obach RS, Baxter JG, Liston TE, Silber BM, Jones BC, MacIntyre F, Rance DJ, and Wastall P (1997) The prediction of human pharmacokinetic parameters from preclinical and in vitro metabolism data. J Pharmacol Exp Ther 283:46-58.

Oie S and Tozer TN (1979) Effect of altered plasma protein binding on apparent volume of distribution. J Pharm Sci 68:1203-1205.

Palkama VJ, Ahonen J, Neuvonen PJ, and Olkkola KT (1999) Effect of saquinavir on the pharmacokinetics and pharmacodynamics of oral and intravenous midazolam. Clin Pharmacol Ther 66:33-39.

Poulin P, Schoenlein K, and Theil FP (2001) Prediction of adipose tissue: plasma partition coefficients for structurally unrelated drugs. J Pharm Sci 90:436-447.

Poulin P and Theil FP (2000) A priori prediction of tissue:plasma partition coefficients of drugs to facilitate the use of physiologically-based pharmacokinetic models in drug discovery. $J$ Pharm Sci 89:16-35.

Poulin P and Theil FP (2002) Prediction of pharmacokinetics prior to in vivo studies. 1. Mechanism-based prediction of volume of distribution. J Pharm Sci 91:129-156.

Poulin P and Theil FP (2009) Development of a novel method for predicting human volume of distribution at steady-state of basic drugs and comparative assessment with existing methods. $J$ Pharm Sci 98:4941-4961.

Rao M, Shiledar R, Bhosale M, Garud M, and Medhekar G (2012) Comparative dissolution studies of marketed tablets of telmisartan in biorelevant media. Am J PharmTech Res 2:654-662.

Rodgers T, Leahy D, and Rowland M (2005) Physiologically based pharmacokinetic modeling 1: predicting the tissue distribution of moderate-to-strong bases. J Pharm Sci 94:1259-1276.

Rodgers T and Rowland M (2006) Physiologically based pharmacokinetic modelling 2: predicting the tissue distribution of acids, very weak bases, neutrals and zwitterions. J Pharm Sci 95:1238-1257.

Rodgers T and Rowland M (2007) Mechanistic approaches to volume of distribution predictions: understanding the processes. Pharm Res 24:918-933.

Rowland M, Peck C, and Tucker G (2011) Physiologically-based pharmacokinetics in drug development and regulatory science. Annu Rev Pharmacol Toxicol 51:45-73.

Sui X, Sun J, Wu X, Li H, Liu J, and He Z (2008) Predicting the volume of distribution of drugs in humans. Curr Drug Metab 9:574-580.

Tannergren C, Bergendal A, Lennernäs H, and Abrahamsson B (2009) Toward an increased understanding of the barriers to colonic drug absorption in humans: implications for early controlled release candidate assessment. Mol Pharm 6:60-73.

van Hoogdalem EJ, Soeishi Y, Matsushima H, and Higuchi S (1997) Disposition of the selective alpha1A-adrenoceptor antagonist tamsulosin in humans: comparison with data from interspecies scaling. J Pharm Sci 86:1156-1161.

Vincent J, Elliott HL, Meredith PA, and Reid JL (1983) Doxazosin, an alpha 1-adrenoceptor antagonist: pharmacokinetics and concentration-effect relationships in man. Br J Clin Pharmacol 15:719-725.

Weber C, Schmitt R, Birnboeck H, Hopfgartner G, van Marle SP, Peeters PA, Jonkman JH, and Jones CR (1996) Pharmacokinetics and pharmacodynamics of the endothelin-receptor antagonist bosentan in healthy human subjects. Clin Pharmacol Ther 60:124-137.

Wu CY and Benet LZ (2005) Predicting drug disposition via application of BCS: transport/ absorption/ elimination interplay and development of a biopharmaceutics drug disposition classification system. Pharm Res 22:11-23.

Address correspondence to: Hidetoshi Shimizu, Mitsubishi Tanabe Pharma Corporation, 2-2-50 Kawagishi, Toda, Saitama 335-8505, Japan. E-mail: shimizu. hidetoshi@ma.mt-pharma.co.jp; orDr. Hiroshi Yamazaki, Showa Pharmaceutical University, 3-3165 Higashi-tamagawa Gakuen, Machida, Tokyo 194-8543, Japan. E-mail: hyamazak@ac.shoyaku.ac.jp. 